\title{
ÉTICA DE LA EMPRESA Y ESTRATEGIAS EMPRESARIALES
}

\author{
Ubaldo Comite
}

Universidad de Calabria

\begin{abstract}
Resumen: El tema de la ética afecta al mundo de las organizaciones productivas, y se propone valorar su compatibilidad con la creación del valor, entendida como mayor producción obtenida respecto al valor de los recursos empleados, objetivo final de cualquier ente productivo. La ética de la empresa significa un verdadero estímulo para las reflexiones sobre la delicada relación empresa - entorno, caracterizada por la necesidad de elaborar estructuras normativas y modelos de reflexión en los que basar las políticas, la gestión y el comportamiento comunes.

Ante estas evidencias, está claro que es oportuno dar una nueva orientación a la cultura empresarial, para ir más allá de la mera venta del producto, situando al cliente en el centro de la organización, pasando de ser una organización tecnology driver a ser customer focused. Se trata de un desafío que en estos años han afrontado muchas organizaciones, pero sin ir más allá de la enunciación de los principios. En efecto, la mayor dificultad reside en concretar los principios en comportamientos eficaces y difusos. La satisfacción del cliente sólo es el punto de partida para la construcción de una relación estable, pero no es suficiente, mientras que es esencial focalizar la atención en un factor que ciertament influye en el valor global de la empresa: la fidelidad de los clientes (customer loyalty).

En este estudio, se analiza el caso de la fidelity card en la experiencia italiana, considerada una herramienta (canal), a través de la cual la empresa puede actuar sobre los elementos subyacentes de la confianza (los llamados driver) y poner en marcha la relación con el cliente final. La tarjeta de fidelidad se convierte a todos los efectos en el vehículo de la relación fiduciaria entre el cliente y el distribuidor. Sin embargo, se trata de una herramienta de una relación fiduciaria sólamente potencial cuando falta una actividad consciente dirigida a explotar las oportunidades emergentes.
\end{abstract}

Palabras clave: ética, consumidor, cliente, empresa, mercado.

\section{Resumo: Ética da empresa e estratexias empresariais}

O tema da ética afecta o mundo das organizacións produtivas, e proponse valorar a súa compatibilidade coa creación do valor, entendida como a maior produción obtida a respecto do valor dos recursos empregados, obxectivo final de calquera proceso produtivo. A ética da empresa significa un verdadeiro estímulo para as reflexións sobre a delicada relación empresacontorno, caracterizada pola necesidade de elaborar estruturas normativas e modelos de reflexión en que basear as políticas, a xestión e o comportamento comúns a todos os actores implicados.

1 Ubaldo Comite, Universidad de Calabria, Via P. Bucci, 87036 Rende (CS), Italia - Docente de Presupuestos y Organización de las Empresas Públicas; comite@unical.it 
Ante estas evidencias, é clara a oportunidade de lle dar unha nova orientación á cultura empresarial, para ir alén da simple venda do produto, e situar o cliente no centro da organización, que deste xeito pasa de ser unha organización technology driver a customer focused. Trátase dun desafío que nestes anos afrontaron moitas organizacións, mais sen superar a enunciación dos principios.

En efecto, a maior dificultade reside en converter os devanditos principios en comportamentos eficaces e difundidos. A satisfacción do cliente só é o punto de partida para construír unha relación estable, mais non é suficiente; así, resulta esencial focalizar a atención nun factor que certamente inflúe no valor global da empresa: a fidelidade dos clientes (customer loyalty).

Neste estudo analízase o caso da creación da tarxeta de fidelidade (fidelity card) na experiencia italiana, considerada unha ferramenta, unha canle, a través da cal a empresa pode actuar sobre os elementos subxacentes da confianza (os chamados drivers) e poñer en marcha a relación co cliente final. A tarxeta de fidelidade convértese para todos os efectos no vehículo da relación fiduciaria entre o cliente e o distribuidor; non obstante, esta relación fiduciaria é soamente potencial cando falta unha actividade consciente dirixida a explotar as oportunidades emerxentes.

Palabras clave: ética, consumidor, cliente, empresa, mercado.

Abstract: Company ethics and business strategies.

The theme of ethics affects the world of productive organisations. This work aims to assess its compatibility with value creation, understood as greater production obtained with respect to the value of the resources used, which is the ultimate objective of any productive entity. Business ethics involve a true stimulus for reflection on the delicate business-environment relationship, characterised by the need to elaborate regulatory structures and models for reflection on which to base common policies, management and behaviour.

Faced with this evidence, it is clear that now is a perfect time to give a new orientation to business culture, in order to go beyond the simple sale of the product, placing the customer at the centre of the organisation, moving from being a technology driver organisation to being customer focused. This is a challenge which many organisations have faced in recent years, but without going beyond stating the principles. Indeed, the greatest difficulty lies in converting the principles into effective and varied behaviour. Customer satisfaction is only the starting point for building a stable relationship, but it is not enough. It is essential to focus attention on a factor which truly influences the company's overall value: customer loyalty.

This study analyses the case of the fidelity card in Italy, considered as a tool (channel), through which the company can act on the underlying elements of confidence (the so-called driver) and begin the relationship with the final customer. The fidelity card becomes for all purposes the vehicle in the trust relationship between the customer and the distributor. However, it is a tool of only a potential trust relationship when conscious activity aimed at exploiting the emerging opportunities is missing.

Key words: ethics, consumer, customer, business, market.

\section{PREMISAS}

El contexto empresarial ha cambiado mucho en los últimos años. El tema de la ética afecta al mundo de las organizaciones productivas, y tiene como objetivo valorar su capacidad de ser compatible con la creación del valor, entendida como mayor producción conseguida respecto al valor de los recursos empleadoS, objetivo final de cualquier ente productivo. Como se puede observar, la realidad de la globalización, caracterizada por múltiples contradicciones, produce al mismo tiempo efectos de aceleración y dece- 
leración en el desarrollo de los niveles de eticidad. Los primeros, dependen de que el aumento de la competitividad impone el desarrollo de los temas de la calidad en sentido amplio, estableciendo exigencias de cooperación interna y externa, introduciendo obligaciones de transparencia muy por encima de los niveles establecidos en la normativa. Los segundos, en cambio, dependen de que el aumento de la competitividad induce a privilegiar las áreas en las que los procesos de industrialización todavía están en una fase inicial y el contexto socio-económico permite que las organizaciones actúen ignorando gran parte de los comportamientos éticos que, sin embargo, constituyen un objetivo que ya se ha alcanzado en la realidad productiva de los países más avanzados. Es importante señalar que no es el beneficio individual el que automáticamente permite la satisfacción del interés colectivo, sino que es el contexto socio-económico el que estimula e impone comportamientos éticos que, como tales, están orientados hacia el interés colectivo, con un impacto directo o indirecto sobre las necesidades. Por lo tanto, es necesario adoptar un punto de vista innovador, caracterizado por la implicación de la empresa en un proceso que tiene que tener como objetivo un nuevo modo de actuar y de pensar.

\section{LA EMPRESA Y SU CONTEXTO DE REFERENCIA}

La empresa, considerada como ente económico destinado a durar en el tiempo (Zappa, 1956) $)^{2}$, es por su naturaleza un fenómeno en continua evolución pero, siendo un sistema abierto, dinámico y probabilístico, hay que analizarla teniendo en cuenta el contexto en el que opera, en relación con el mercado y con el conjunto de los actores económicos que interaccionan con ella.

Por lo tanto, la empresa puede asumir diferentes características, complementarias y no alternativas. La empresa es abierta, ya que propone continuos intercambios con el exterior; dinámica, ya que cambia en el tiempo y en el espacio (Bertini, 1975); compleja, puesto que los elementos y las relaciones que la componen son múltiples; finalística, ya que tiene la capacidad de lograr un resultado; probabilística, porque su funcionamiento está sujeto a incertidumbres y riesgos (Ferrarsi Franceschi, 1984; Fiume, 2000; Giunta, 1995).

Además, el sistema empresa forma parte de un contexto de orden superior, representado por el ámbito socio-económico. Ese ámbito, está formado por otros subsistemas, constituidos por las categorías lógicas identificadas para circunscribir el análisis a aspectos preestablecidos (Catturi, 2003): el sector, el mercado, el área geográfica, el distrito, son posibles sistemas.

Indudablemente, el escenario actual es completante diferente al de hace unos años, debido a que las empresas tienen que afrontar un nivel de presión competitiva desconocido hasta hace poco (Castaldo y Bertozzi, 2000), también como resultado de la progre-

2 "La empresa es un ente económico destinado a durar que, para la satisfacción de las necesidades humanas ordena y desarrolla la coordinación, la producción o el abastecimiento y el consumo de la riqueza de forma continua" (cfr. Zappa, 1956). 
siva globalización de los mercados, que imponen al empresario respuestas rápidas y adecuadas (Gazzoni 2003) ${ }^{3}$, para contrastar formas competitivas cada vez más fuertes. Esta problemática afecta indistintamente a todas las empresas, pequeñas, medianas y grandes, que diariamente se ven obligadas a afrontar el mercado y los diferentes competidores (Fazzini, 2006). Para afrontar un escenario tan delicado, es necesario ofrecer productos y servicios capaces de garantizar un valor añadido a medio - largo plazo, predisponiendo un abanico de alternativas cada vez más amplio en términos de servicios ofrecidos a la clientela, con la consecuencia de ejercer lo que en lenguaje empresarial se conoce como fidelización (Anselmi y Lattanzi, 2002).

El dato inicial ya no es el producto, sino las necesidades y las exigencias del consumidor. Se pasa del concepto de "vender lo que se es capaz de producir", al de "producir lo que se es capaz de vender" (Volpato, 2000). Las empresas han comprendido que, en primer lugar, deben conocer las necesidades y los deseos de los consumidores, para así ofrecerles un producto capaz de satisfacerles. En tales contextos, satisfacer al cliente mejor que los competidores es una fuente de ventajas competitivas para la empresa (Invernizzi, 1999).

\section{LA IMPORTANCIA DE LOS VALORES ÉTICOS EN LA EMPRESA}

La empresa es un ente cultural a todos los efectos, y los empresarios están asumiendo plenamente conciencia de ello, teniendo cada vez más clara la visión y la importancia de estos valores, con las consiguientes implicaciones sociales. Ética y eficiencia no son eslogans, sino dos directrices en las que inspirar la programación y la actividad de empresa, hasta convertirlas en modelos de comportamiento para toda la sociedad.

Las discusiones sobre la ética en la sociedad de consumo (Cavalieri, 2002), y más aún, en el mundo de los negocios, en estos últimos tiempos están muy de actualidad, como si existiese un sentimiento común difundido para recuperar en las elecciones políticas y sociales, en el mundo del trabajo, en las acciones de cada día, contenidos de moralidad y justicia más profundos, que el dinamismo y la conflictividad de la vida cotidiana parecen haber borrado, ofuscado o, por lo menos, rodeado de profundas incertidumbres.

3 Según el art. 2082 c.c. quien ejerce profesionalmente una actividad económica organizada con el objetivo de la producción o del intercambio de bienes y servicios es un empresario.

La actividad empresarial, por tanto, la definen las siguientes características:

a) Actividad económica. La empresa tiene como objetivo producir nueva riqueza. Por tanto, no lo sería la actividad consistente en el mero disfrute de bienes.

b) Producción e intercambio de bienes y servicios. No sólo la producción de bienes constituye nueva riqueza, sino también su intercambio, ya que el bien, una vez distribuido a los consumidores, aumenta la utilidad pasada.

c) Profesionalidad. Es empresario sólo quien desarrolla la actividad económica de forma estable y no ocasional, mientras que no es necesario que también tenga carácter continuativo.

d) Organización. La actividad de organización encierra la esencia del fenómeno empresarial. Términos de referencia objetivo de la organización son el capital y el trabajo.

e) Aunque una parte, en realidad minoritaria de la Doctrina, ha intentado demostrar su admisibilidad en nuestro ordenamiento, no es posible desarrollar la actividad empresarial de forma secreta (cfr. Gazzoni, 2003). 
Se sabe que la empresa actual opera en condiciones ambientales inciertas y difíciles. En efecto, el grado de complejidad de los fenómenos socio-económicos parece que ha aumentado innegablemente, y tal sensación parece legítima tanto en sentido cuantitativo, por el gran número de interlocutores implicados ${ }^{4}$ por y en la actividad empresarial, como en sentido espacial, por las correlaciones existentes entre áreas geográficas lejanas entre sí, como en términos de dimensiones, en relación con las múltiples esferas culturales, políticas etc.

Esto se debe a que las empresas no sobreviven ni se desarrollan si no están en sintonía con las dinámicas del sistema de pertenencia a cuya instancia, a cuyos movimientos, necesitan dedicar una atención constante, además de, obviamente, a la estructura del control empresarial, a las relaciones humanas, a las tareas laborales, a la tecnología y a la innovación, a la calidad, a los roles familiares, a la formación cultural juvenil, al contexto cívico, al entorno institucional, a los medios de comunicación, a las relaciones entre las naciones y a la ecología.

Por lo tanto, es necesario aplicar un método que responda a las instancias culturales descritas y a las condiciones éticas. Este punto de vista innovador estará caracterizado por la implicación de la empresa en un proceso que debería tener el objetivo de abrir nuevos horizontes en su forma de actuar y de pensar.

Esta nueva dimensión tiene que responder a la exigencia de percibir, comprender, amortizar e interiorizar tensiones, presiones e instancias socio-culturales, dialogando con ellas, de forma que se obtengan resultados positivos.

Esto pone en relieve que la ética está en sinergia con la empresa, para mejorar la convivencia. Entrando en el aspecto económico - empresarial específico, con el término ética se hace referencia: al sistema de los valores de la empresa; a operar en tal sistema para poder definirlo; a la reflexión sobre las elecciones y a la valoración de las alternativas dirigidas a realizar ese conjunto de valores en la unidad económica; a los principios gestionales y a las normas de comportamiento que, delineados en tal reflexión, regulan

4 De hecho, la empresa tiene que verse a sí misma como una entidad que necesita confluir en un amplio contexto de sujetos que interactúan entre ellos, con el objetivo de instaurar, con el mayor número posible de ellos, nuevas relaciones, cada vez más estrechas y rentables. Los interlocutores sociales también se denominan stakeholders. Este término está formado por dos palabras: stake, interés, y holder, posesor, portador.

Por lo tanto, se refiere a los portadores de intereses, aquellos que se juegan algo, e individuos y grupos de individuos que además de los tradicionales posesores de cuotas de capital, pueden: influir en la gestión de una empresa; generando elementos de fortaleza y debilidad de la actividad económica; al mismo tiempo, son los destinatarios inmediatos o mediatos de los actos de empresa; por lo tanto están implicados, directamente o indirectamente, en los efectos económicos, financieros, tecnológicos, sociales, políticos, etc... de la acción económica.

Cada una de las categorías puede individuarse en base a las posiciones, los papeles y las funciones que los citados interlocutores asumen cuando entran en contacto directo o indirecto con una empresa. Por tanto, hay que considerarlos componentes del escenario operativo de la gestión de la empresa .

Surge así la exigencia, por parte de la empresa, de dedicar recursos intelectuales, tiempo y sensibilidad a la gestián de las relaciones con los stakeholders, desarrollando con ellos flujos de comunicación, procesos de coordinación y motivos de colaboración. Todo esto, hace que la empresa dé un salto de calidad, ya que, en sustancia, la interiorización del concepto de stakeholder debe permitir el desarrollo de una sensibilidad ética y social especial. 
los actos operativos del organismo empresarial, manifestando su cultura ética y permitiéndole perseguir la realización del sistema de valores escogido; al control global sobre los actos de la empresa, es decir, a la valoración de la correspondencia de las acciones emprendidas con el sistema de valores guía en el caso de que se perdiera la sintonía con los valores difundidos entre los stakeholders ${ }^{5}$.

Por tanto, se trata, de asumir un sistema de valores que sirva como punto de referencia para las decisiones y los comportamientos de empresa y que, a través de oportunos esquemas de reflexión sobre la gestión, ofrezca criterios guía para la conducta de los órganos empresariales.

En consecuencia, la ética desarrolla la labor de herramienta o proceso de orientación de la gestión, a través de una función de problem-solving ${ }^{6}$.

Por tanto, la empresa ética es un lugar en el que los principios de un derecho natural pueden conjugarse con los principios de eficacia y eficiencia que tienen que caracterizar la cotidianidad de la empresa, con la elección de valores compartidos, que se fortalecen y se potencian en la retroalimentación de un bienestar cualitativo, es decir, en la búsqueda de una elevada calidad de la vida, que no se puede conseguir sin la sinergia del grupo ${ }^{7}$.

La empresa ética actúa de forma que el consumidor - cliente manifieste sus exigencias, a través del proceso definido de learning organization, que permite desarrollar, a través del intercambio, el propio proceso de concienciación.

En una situación similar, en la que se puede desarrollar una teoría de los juegos de colaboración que van desde cero hasta el nivel positivo, se puede hablar de búsqueda compartida de un valor ético que puede iluminar la vision de la empresa y permitir el refuerzo de los sistemas de convicciones de quienes entran en contacto con tal organización (Figura 1).

De esta forma, la empresa también asume una función social de vehículo de unos valores actualmente considerados muy necesarios, contribuyendo, junto con las institucio-

5 Los stakeholder se dividen en tres grupos, en base a la proximidad a la empresa:

1) stakeholder secundarios que representan a la comunidad en el sentido más amplio. Va desde los entes privados hasta el ámbito internacional, pasando por los grupos de presión;

2) stakeholder primarios externos que representan a grupos de interés. Están directamente relacionados con la empresa, pero son externos;

3) stakeholder primarios internos, que son los socios, los trabajadores, los voluntarios de las empresas sin ánimo de lucro, es decir, quienes tienen un interés (stake) dentro de la estructura empresarial.

$6 \mathrm{La}$ ética puede definirse como un modelo de comportamiento que privilegia los valores morales de la honradez, la justicia (equidad) y el respeto de la personalidad humana. Los valores éticos esenciales son honradez, integridad, fiabilidad, fidelidad, equidad, altruismo.

7 No hay que confundir el concepto de ética con el respeto de la ley y, por lo tanto, como disuasión contra las violaciones de normas del derecho; tiene que considerarse un modelo de comportamiento basado en los valores de la equidad, la lealtad y la fiabilidad. En otras palabras, haciendo especial referencia al mundo empresarial, no se pueden configurar como "éticas" las decisiones para, por ejemplo, garantizar la sanidad de los productos, la seguridad en las fábricas, evitar la dispersión de substancias tóxicas en el medio ambiente. Si estos elemento no se respetan, no se trata de elementos que no son éticos, sino delictivos, sancionados por la ley y contrarios a la moral. 
nes dedicadas al crecimiento y al desarrollo colectivo, a crear un modelo a seguir para una sociedad más adecuada a las exigencias de bienestar que no esté caracterizada exclusivamente por el énfasis en los bienes, sino también en las relaciones humanas y en los valores.

Figura 1 - La empresa y los interlocutores interesados en ella (valores en \%).

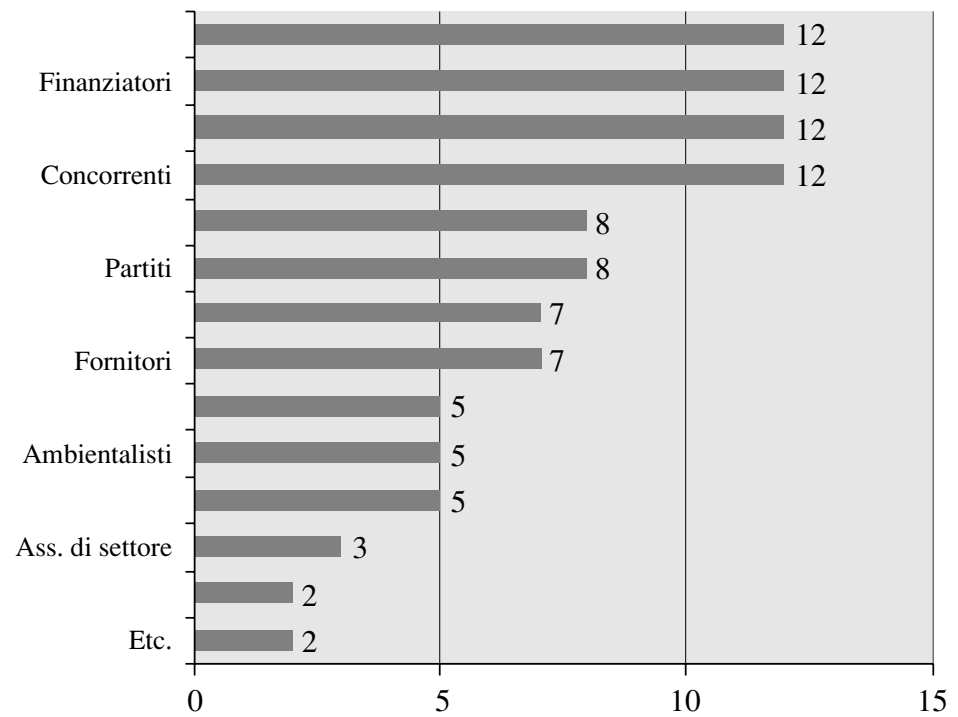

Fuente: elaboración propia

El tema de la ética se cruza con la tradicional antinomia ética-beneficio, típica exclusivamente del mundo de las empresas, para incluir la creación del valor, que también se abre a las lógicas gestionales de las administraciones públicas, de las cooperativas, de las asociaciones y de las fundaciones.

Eso se debe al progreso tecnológico, la globalización y la evolución de las relaciones entre la producción y el consumo, que han determinado una profunda y rápida resolución en los sistemas económicos y en los comportamientos de las organizaciones productivas que operan en ellas.

El cambio ha sido radical, y entre las temáticas importantes en función de los objetivos del discurso sobre la ética hallamos:

1. el tema de la transparencia, que tiende a convertirse en una condición esencial de acceso y permanencia en los mercados, sobre todo financieros, en los que los recursos se orientan cada vez más hacia las empresas con mayor rendimiento, pero que también imponen las Authorities como exigencia de control y salvaguardia de algunos intereses generales;

2. el tema de la calidad, en cuyo ámbito se incluyen, no sólo las exigencias de calidad (en sentido estricto) de la producción final, orientadas a la satisfacción de 
las expectativas específicas del cliente, sino además: las exigencias de calidad (en sentido amplio) dentro de las diferentes fases del proceso productivo, en la filosofía del Total Quality Management; la tendencia a seguir lógicas de producción ecocompatibles con la salvaguardia medioambiental; la tendencia de la producción a asumir cada vez más la configuración de servicios de alto valor, implicando en la responsabilidad del diseño productivo a proveedores y clientes, en una lógica de integración que transforma una economía basada en los intercambios, en una economía basada en la colaboración sistemática entre diferentes sujetos en la cadena de creación del valor;

3. el tema de la cooperación, seguramente el más sugestivo, porque abre el camino a las diferentes formas de contacto, colaboración, alianzas entre organizaciones productivas de cualquier naturaleza jurídica y dimensión, que, antes, vivían autónomamente su experiencia productiva, mientras que hoy están obligadas a buscar niveles de conocimiento adecuados, participando en sistemas más amplios, de los que obtienen una parte no desdeñable de los recursos materiales e inmateriales necesarios para realizar la combinación productiva y sustentar la innovación, para así competir con éxito y con posibilidades de supervivencia en el mercado global. Este tema, además, incluye las dinámicas organizativas internas, transformando lentamente relaciones basadas en formas jerárquicas, en relaciones mucho más flexibles, basadas en la participación y la responsabilización, que requieren las formas de descentralización estratégica más extremas.

Es evidente que la tendencia hacia la rectitud y la transparencia, así como la exigencia de calidad en la producción y la responsabilización respecto a la salvaguardia medioambiental, actualmente son condiciones necesarias para competir, y entrañan importantes contenidos éticos y valencias sociales.

Todavía más significativos, aunque menos evidentes, son los contenidos éticos de las diferentes formas de cooperación externa entre organizaciones productivas, o dentro de la misma organización.

En efecto:

- reemplazar las lógicas del intercambio y la competencia con una lógica de colaboración sistemática entre las partes interesadas en la relación, significa sustituir los limitados contenidos éticos de las relaciones contractuales, con el imprevisible potencial ético del diálogo y el intercambio de conocimientos;

- completar las tradicionales reglas de la jerarquía con una lógica diferente basada en la participación, no sólo significa aumentar la eficiencia, sino también elevar el contenido de eticidad en las relaciones laborales, hacia el desarrollo de la capacidad creativa, y hacia la realización del individuo y la adquisición de mayor autonomía y responsabilidad.

Por lo tanto, podemos constatar que el contexto socio-económico estimula a las unidades productivas a la competición a través de comportamientos que incorporan niveles de eticidad crecientes, e impone la adquisición de responsabilidades cada vez más complejas, debido a que ya no están dirigidas solamente a algunas categorías de 
sujetos, sino que también incluyen a los accionistas, a los diferentes stakeholders y a toda la sociedad.

Para actuar conscientemente, la ética tiene que conocer, sobre el análisis económico-empresarial:

- la relación entre los eventos económicos;

- el valor y el grado de compatibilidad entre los diferentes objetivos a alcanzar;

- las consecuencias económicas de la acción orientada a algunos objetivos;

- la conveniencia o la inoportunidad de crear datos objetivos.

Además, las disciplinas económico-empresariales pueden aclarar las evoluciones:

- en la función económica de ciertos instrumentos, como por ejemplo: riqueza y capital;

- en la estructura y en la dirección de la empresa, por ejemplo unión o distinción entre la propiedad y el gobierno;

En consecuencia, la ética puede modificar a lo largo del tiempo sus valoraciones sobre el modus agendi del individuo, y sobre la magnitud del beneficio de empresa.

\section{LA RELACIÓN EMPRESA-RSE EN ITALIA}

La RSE, en Italia, está pasando de la simple experimentación de lo que inicialmente pudo ser una "moda directiva", a un nuevo modo de trabajar y hacer empresa ${ }^{8}$ (Tabla 1).

Tabla 1 - Inversiones en RSE.

Nuevas inversiones en RSE (tipologías)

$\%$ de empresas

\begin{tabular}{ll} 
Balance social/Balance de sostenibilidad & 54,0 \\
\hline Código ético & 52,4 \\
\hline Certificado & 50,8 \\
\hline Actividad de verificación & 39,7 \\
\hline Realización de nuevos productos & 38,1 \\
\hline Otro & 34,9 \\
\hline
\end{tabular}

Fuente: Observatorio Istud-Dnv

8 Es lo que se deduce de la investigación "La CSR in Italia: dalla sperimentazione al consolidamento"("La RSE en Italia: de la experimentación a la consolidación"), realizada por el Observatorio Istud-Dnv. Un estudio que ha durado diez meses y que ha analizado 80 grandes empresas nacionales y multinacionales, ha permitido individuar un "núcleo duro" de empresas para las cuales la inversión en la responsabilidad social ya es o va a ser, una prioridad estratégica. En el ámbito de la RSE, esto lleva a privilegiar las prácticas más evidentes y formalizadas: códigos éticos, certificados, uso más consciente del balance social. 
En este sentido, es posible representarla por estadios evolutivos:

- la RSE informal. La mayor parte de las empresas italianas, empezando por las de pequeñas dimensiones, se encuentra en este estadio. El tema de la RSE todavía no se afronta de forma consciente y formalizada. Sin embargo, eso no significa que las empresas descuiden las relaciones con los interlocutores sociales;

- la RSE actual. Debido al impulso de las crecientes expectativas sociales y medioambientales, las empresas que operan en Italia, sobre todo las de grandes dimensiones, han iniciado con los “clásicos” del repertorio de la RSE: el código ético ${ }^{9}$, el balance social o de sostenibilidad ${ }^{10}$, los certificados ambientales y de

9 Las razones por las que el mundo empresarial debería declararse a favor de los códigos éticos se deben a: - desde el punto de vista del manager, el código ético: aclara eventuales márgenes de incertidumbre en las políticas empresariales; ayuda a resolver dilemas éticos; ayuda a rechazar peticiones irrazonables, a resistir a las presiones de los superiores y de los subordinados; ayuda a negar favores especiales a clientes o categorías de clientes; sirve como guía en situaciones de despidos y cancelación de contratos a proveedores; favorece el sentido satisfacción que deriva de la conciencia de trabajar en un contexto que atribuye a la honradez moral su justo valor.

- desde el punto de vista de la empresa, el código ético: elimina prácticas incorrectas que provocan la pérdida de clientes y pérdidas económicas a corto plazo: elimina prácticas indeseadas que van despojando a la empresa de la benevolencia de la clientela, los dependientes, los proveedores, los accionistas, el gobierno, la sociedad, con la posibilidad de registrar pérdidas económicas a largo plazo; facilita la adopción de nuevas prácticas; aclara dudas sobre la ética de las políticas de empresa; ayuda a los dirigentes a resolver dilemas éticos actuando al mismo tiempo en beneficio de la empresa; exime parcialmente a los dirigentes de las dificultades a la hora de tomar decisiones graves cuando no hay tiempo suficiente para evaluar minuciosamente todas las implicaciones del caso; ofrece modalidades de conducta alternativas respecto a la introducción de nuevas reglas en los mecanismos de motivación y de incentivación existentes, sobre todo en los casos en que los mecanismos de investigación y las sanciones necesarias para hacer respetar las reglas sean demasiado onerosos; impide que los dirigentes exploten al personal; facilita la lucha contra los dependientes que violan el código o la ley, por ejemplo aceptando sobornos; reduce el uso de medios coercitivos a la hora de detectar delitos que, al oponerse a valores de orden superior, hunden la moral del personal.

- desde el punto de vista sectorial, el código ético: previene la reglamentación gubernamental, que se pondría en marcha si la empresa no se hubiese dotado de una autorregulación; erradica prácticas, por ejemplo tangentes sobre ventas en el extranjero, en las que todos los sujetos implicados sufren pérdidas.

- desde el punto de vista del sistema empresarial, el código ético: contribuye a restablecer la confianza del público en el mundo de los negocios. Las continuas revelaciondes de hechos contrarios a la ética perjudican la reputación del mundo de los negocios ante el público y debilitan su apoyo al sistema de libre mercado, provocan continuas peticiones de reglamentación gubernamental y el paso a un sistema de propiedad pública, minando la voluntad de resistencia antre otras amenazas, internas y externas, para el sistema (cfr. Riolo F., Etica degli affari e codici aziendali, Edibank, Milano, 1995).

10 Balance social es el tèrmino que se utiliza comúnmente para hacer referencia a la herramienta de monitorización, rendición de cuentas y comunicación del proceso de gestión responsable puesto en marcha por una organización, que da a conocer e ilustra el impacto de las actuaciones medioambientales, sociales y económicas y la relación que se instaura entre éstas y las estrategias y los resultados empresariales. El balance social tiene como objetivo primario la representación de los valores y los efectos que la actividad de la empresa provoca en el contexto y en el conjunto de stakeholders. Normalmente elabora previsiones para la fase sucesiva y adopta una orientación similar a la utilizada para determinar el valor añadido. La denominación "balance social" es convencional e incluye varias herramientas, a veces muy diferentes entre sí. Muy a menudo, los términos balance social y balance de sostenibilidad se utilizan como sinónimos. Pero enfatizar la sostenibilidad, implica una evolución en el proceso de rendición de cuentas y comunicación responsabile, ya que dirige la atención hacia el futuro. De hecho, el desarrollo sostenible se define como aquél que no compromete las posibilidades de crecimiento de las generaciones futuras, preservando la calidad y la cantidad del patrimonio de recursos naturales, que son limitados. Por lo tanto, el objetico de un balance de sostenibilidad es demostrar que 
seguridad. Esta fase presenta un peligro característico, pero también algunas ventajas potenciales. El peligro reside en la posibilidad de que la adopción de estas prácticas puede limitarse al mero formalismo. Sin embargo, si se interpretan bien, las prácticas típicas de RSE pueden contribuir a la evolución de la empresa;

- la RSE sistemática. Si la fase de la RSE actual se vive fisiológicamente, se crean las condiciones por la transición al estadio siguiente: llega el momento de afrontar concretamente y sistemáticamente las implicaciones socio-ambientales de las actividades empresariales. En el estadio de la RSE sistemática, a menudo, el management utiliza algunas de las herramientas típicas del análisis estratégico, como por ejemplo, el modelo de la cadena de valor. Y también es típico de esta fase proceder a la monitorización de las best practice, además de intensificar el diálogo con los stakeholders para comprender sus expectativas y así poder aplicar una estrategia más eficaz respecto a los mismos. Por consiguiente, en este tercer estadio, surgen muchas iniciativas nuevas: planes formalizados en defensa de la igualdad de oportunidades; articulación de nuevas políticas hacia los consumidores; sistemas de valoración e incentivación de los managers, etc.;

- la RSE innovadora. La familiaridad que el top management ya ha adquirido con los temas de la responsabilidad social puede ser el trampolín de lanzamiento para una ulterior transición hacia una RSE más explícitamente considerada como fuente de ventajas competitivas. En esta fase, la RSE es sinónimo de creatividad al servicio de la satisfacción de las expectativas de los stakeholders. Esta fase se caracteriza por la lógica win to win: ganan los interlocutores sociales (consumidores, comunidad, medio ambiente) y la empresa, que aumenta la facturación y mejora su reputación.

Debido a la creciente exigencia de los stakeholders de que las empresas rindan cuentas sobre sus actuaciones sociales y medioambientales, actualmente, las mismas deben competir en un nuevo frente además del económico y financiero. En consecuencia, el único camino que pueden seguir las empresas es el del desarrollo sostenible, que es una condición necesaria para crear valor a medio-largo plazo. Y ya que el debate sobre la definición de empresa responsable se está desplazando hacia el ámbito del sustainability governance, se tendrán que elaborar estrategias adecuadas rápidamente para contemplar, dentro de lo posible, las exigencias, sobre todo de tipo ético, manifestadas por todas las categorías de stakeholders (Marra, 2002).

\section{LA FIDELIZACIÓN DEL CLIENTE}

Para dar sentido a cualquier intervención sobre estos elementos y poner en práctica el principio de vender soluciones válidas para el cliente, en primer lugar es necesario

el equilibrio entre riqueza, igualdad social y respeto de los ecosistemas es posible. Además, generalmente se caracteriza por la presencia de un presupuesto que contempla los compromisos de mejoramiento para el siguiente periodo. 
saber dónde reside el valor para el cliente. Para difundir comportamientos que garanticen una gestión eficaz de la relación con el cliente, el punto de partida es un conocimiento minucioso de los procesos de adquisición, que hay que profundizar y describir.

Cada vez es más difícil catalogar los comportamientos y tendencias del nuevo consumidor, que parece voluble (pretende productos y servicios siempre nuevos y diferentes), despiadado (ignora a la empresa si ésta no satisface sus deseos), exigente (exige a un empeño continuo por parte de la empresa), infiel (cambia continuamente de proveedor), vengativo (ignora a la empresa proveedora de bienes y servicios si se considera insatisfecho), egocéntrico (pretende ser diferente de los demás, y como tal, pretende un trato personalizado) (Figini, 2003).

Las distinciones entre los diferentes programas de fidelización pueden realizarse en base a muchos elementos. Por ejemplo, existen aquellos más orientados al premio y aquellos más orientados a la relación. En realidad, más allá de las diferentes clasificaciones posibles, los esquemas de los programas de fidelización tienden a parecerse cada vez más, debido a los procesos de imitación aplicados por las empresas competidoras.

De los estudios realizados sobre la clientela se deduce que los programas de fidelización se caracterizan más por un carácter defensivo que ofensivo (Manaresi, 1999). De hecho, emerge que los programas promocionales basados en tecnologías avanzadas, como los relacionados con las tarjetas de fidelidad, no consiguen que la empresa conquiste nuevos clientes, sino que delimitan mejor los confines del propio mercado potencial, logrando atraer con un mayor grado de fidelidad a clientes que ya frecuentan el punto de venta. En otras palabras, las empresas no logran convertir en clientes a individuos que antes de la introducción de la tarjeta de fidelidad frecuentaban, para la mayor parte de su compra, otros establecimientos. En cambio, los programas de fidelidad, actuando sobre la propia clientela, pueden reforzar la relación con estos clientes, valorizando (en términos de facturación y penetración en un mayor número de categorías), los motivos que el cliente ya tiene para ser propenso de forma "natural"a la fidelidad respecto al punto de venta (proximidad y localización favorable, oferta coherente con las necesidades del cliente, servicio de alto nivel). Gracias al programa de fidelidad aumenta, aunque no de forma elevada, el porcentaje de gasto que el cliente que ya es fiel efectúa en el punto venta; en el cliente disminuye la propensión "a la infidelidad ocasional".

Por lo tanto, los programas de fidelización se configuran como intervenciones defensivas respecto a los competidores, salvaguardando la cuota de mercado de la empresa, reforzando las costumbres y creando elementos de fidelidad.

Por tanto, podemos afirmar que la fidelidad es un concepto complejo, formado por dos dimensiones, (Lugli y Pellegrini, 2002): la dimensión cognitiva y la comportamental.

La fidelidad comportamental consiste en la repetición de la adquisición de la misma alternativa de oferta por parte del cliente; sin embargo, eso no es suficiente para poder afirmar que ese cliente es efectivamente fiel. En efecto, existen situaciones en las que la fidelidad comportamental no está acompañada por el aspecto cognitivo, consistente en el reconocimiento consciente por parte del cliente del hecho que la empresa 
satisface plenamente sus necesidades. Los comportamientos repetitivos se verifican en general, en presencia de:

- fidelidad al monopolista: cuando el proveedor del bien o servicio es único, en este caso, el cliente es fiel por necesidad;

- fidelidad por inercia: existen alternativas de adquisición, pero el cliente no es consciente de ello, o bien no busca alternativas porque cree que la actividad de búsqueda es demasiado onerosa, en términos de esfuerzo económico y tiempo;

- fidelidad por proximidad: típica de la distribución comercial, es parecida a la del monopolista, y se caracteriza por el ser inversamente proporcional a la distancia del cliente respecto al proveedor;

- fidelidad utilitarista: el cliente repite las adquisiciones hasta que tiene una conveniencia en el precio, pero cambia en el momento en que un competidor ofrece una alternativa más ventajosa;

- fidelidad incentivada: se difunde con la introducción de programas de fidelidad y otras herramientas de fidelización, cada vez más adoptadas por las empresas. Éstas recompensan con descuentos, premios, privilegios y servicios, a los clientes que repiten sus adquisiciones.

Este tipo de fidelidad presenta el riesgo de transformarse en fidelidad utilitarista: los clientes que desean gozar de los beneficios prometidos se hacen fieles por este motivo, pero pasarán fácilmente a la competencia cuando ésta proponga privilegios mayores o esfuerzos menores para conseguir las mismas ventajas.

A diferencia de la fidelidad comportamental, la cognitiva deriva de un acto de voluntad preciso del cliente, que reconoce la correspondencia de la oferta respecto a sus necesidades.

Por consiguiente, la fidelidad cognitiva es el único tipo de fidelidad sobre la que la empresa puede contar como ventaja competitiva duradera. Se trata de una alineación de los valores de la empresa con los del consumidor, que les permite resistir a la fuerza de los competidores y atenúa el impacto de eventuales experiencias negativas en el comportamiento adquisitivo de los clientes. El cliente que muestra este tipo de fidelidad, repite sus adquisiciones libre de constricciones.

Los programas de fidelización, gracias al empleo de la fidelity card, permiten conocer de forma más detallada las exigencias específicas de cada cliente, sus costumbres de adquisición, las modalidades y los tiempos de consumo, proporcionando información detallada sobre la percepción del valor que el cliente atribuye a algunos elementos de la oferta total, permitiendo así que el retailer aplique las acciones comerciales apropiadas en función de las preferencias expresadas y manifestadas por los clientes.

Los límites en el desarrollo de las tarjetas de fidelidad residen en la incapacidad o ineficiencia de la empresa a la hora de analizar correctamente los datos procedentes de las tarjetas. Las bases de datos sobre los clientes tienen que ser analizadas y explotadas adecuadamente. Gracias a los programas de fidelidad, la empresa puede obtener una gran cantidad de información sobre el comportamiento del cliente. 
Por lo que se refiere al desarrollo de las tarjetas de fidelización, es posible identificar, a nivel general, algunos estadios evolutivos en los que se manifiestan estrategias de lanzamiento, gestión y desarrollo, y que representan diferentes steps progresivos.

Las fases del desarrollo pueden analizarse considerando los diferentes objetivos que las empresas definen en cada step, o sea, el objetivo que desean alcanzar y el segmento de referencia para cada actividad desarrollada; las herramientas operativas utilizadas para lograr los objetivos establecidos; las actividades promocionales propuestas.

El objetivo general que se quiere alcanzar en la fase de lanzamiento de un programa de fidelización relacionado con una tarjeta de fidelidad, es obtener, en primer lugar, un crecimiento de la base de datos sobre los clientes y, en segundo lugar, un aumento de la fidelidad comportamental de la clientela, puesto que el papel y las iniciativas promocionales ofrecidas a los poseedores deberían proporcionar al consumidor un incentivo a seguir eligiendo el mismo punto de venta para efectuar las mismas adquisiciones y, al mismo tiempo, incrementar la frecuencia de las visitas, de forma que se puedan acumular más puntos. Tal objetivo se puede definir como "conveniencia o promoción", y generalmente se realiza a través de la introducción de la tarjeta de fidelidad. En efecto, ésta última no ofrece servicios particulares de pago o financieros, pero garantiza al consumidor la oportunidad de acceder a las promociones reservadas a los titulares de la tarjeta y acumular puntos útiles para la obtención de cupones o la adquisición de productos o servicios con condiciones ventajosas. Las estrategias promocionales utilizadas para alcanzar este objetivo son las tradicionales promociones de continuidad, cuyo objetivo fundamental es inducir al consumidor a un comportamiento fiel a la marca, sin dejar de actuar directamente para el desarrollo de una relación fiduciaria consciente respecto a la marca.

La fase siguiente a la introducción de la tarjeta es analizar los datos recogidos para utilizarlos más adelante en la definición de estrategias específicas. El objetivo general de esta segunda fase es seleccionar diferentes modalidades de gestión de la clientela y profundizar la relación, tanto a través del análisis de los comportamientos de adquisición, como del nivel de rentabilidad de los diferentes segmentos. Generalmente, esta fase se caracteriza por la introducción de nuevas tarjetas de fidelidad diferenciadas por tipologías de consumidores, agrupadas en base a características socio-demográficas o comportamentales.

En la tercera fase se consolida la relación que corresponde al estadio fiduciario del intercambio (Castaldo y Cillo, 2001).

En este caso, el objetivo principal es ampliar la relación con la clientela, aumentando las oportunidades y las modalidades de contacto entre el punto de venta y el consumidor, también con el objetivo de ampliar las oportunidades de business que se abren para el distribuidor mismo o para el desarrollo de acuerdos con otras empresas.

Por lo tanto, es posible sintetizar la evolución de las estrategias de loyalty management, junto con las tarjetas de fidelidad, como un itinerario evolutivo en cuatro fases, en las que la confianza puede ser conquistada de forma secuencial a través de: la puesta en marcha de iniciativas diferenciadas para cada segmento de consumidores; servicios añadidos (crédito) y programas de loyalty management desarrollado; el incremento de servicios a través de la implicación de sujetos externos. 


\section{CONCLUSIONES}

La ética de empresa constituye un nuevo estímulo para las reflexiones sobre la delicada relación empresa - entorno, caracterizada por la necesidad de elaborar plataformas normativas y modelos de reflexión sobre los que fundar las políticas, la gestión y el comportamiento comunes.

Por tanto, tales plataformas deberían incluir un sistema de valores declarado y promovido explícitamente por las empresas, reconocido como punto de referencia para el desarrollo de reflexiones, análisis y valoraciones éticas sobre los objetivos, los planes y las alternativas de la gestión.

A tal propósito, se puede afirmar que cuando la ética se considera una reflexión continua sobre el significado de las acciones, está claro que las personas y los organismos económicos tienen que ser conscientes de que están desarrollando constantemente tal reflexión.

Por lo tanto, el aspecto más relevante reside en el estímulo a expresar una opinión respecto a los comportamientos de las empresas, siendo conscientes del hecho que, en cualquier proceso empresarial, es necesario descubrir y desarrollar una dimensión ética.

El análisis ético manifiesta su mayor potencial cuando esa opinión se basa en la evidencia de cómo esos comportamientos afrontan y satisfacen las expectativas éticas de la sociedad.

Se descubre así la base de la valoración social del comportamiento de la empresa en la medición del grado de relación entre los efectos sociales de sus comportamientos gestionales y las expectativas e instancias procedentes del entorno externo.

Por tanto, el comportamiento ético de la empresa depende del grado de sintonía y convergencia entre: a) valores de empresa que rigen las decisiones gestionales; b) valores de la colectividad que interacciona con la unidad económica.

Así, se puede comprender perfectamente cuál es para la empresa el objetivo de su responsabilidad social y el sentido de su sensibilidad social.

Por lo tanto, en una gestión éticamente orientada hacia los valores del entorno social, se encuentra la respuesta unitaria y globalizadora que muchos buscan frente a las instancias y las presiones dirigidas por la colectividad hacia el mundo de los negocios.

En esta respuesta hay que basar las decisiones empresariales capaces de determinar relaciones estables y duraderas con las contrapartes sociales. En efecto, la ética de empresa influye profundamente en la capacidad de: aprovechar las oportunidades; comprender y administrar los recursos reales a disposición; interpretar el entorno social de los stakeholders; controlar y medir los efectos y los resultados de la actividad.

Efectivamente, la ética de empresa permite comprender los valores del entorno social que tienen que ser apreciados e interiorizados para así poder plantear las respuestas oportunas.

De ella derivan, además, las normas de comportamiento a asumir por los operadores de la empresa a la hora de afrontar y dialogar con las posturas, las exigencias y las 
dinámicas de los clientes, de los proveedores, de los sindicatos, de los medios de comunicación, de los entes locales, etc... Al mismo tiempo, de la conciencia ética de la empresa depende el clima de las relaciones internas entre los dependientes, los directivos y los socios de la empresa.

En efecto, cada empresa actúa en relación a sus creencias, es decir, en función de los elementos que para ella tienen un valor, o, en otras palabras, en relación con la ética que en todo caso posee, aunque sea de forma inconsciente.

En resumen, cada empresa se caracteriza por un sistema de valores propio y por una cierta forma de aplicarlo, a partir del que se definen los objetivos y las normas de su interacción con la comunidad social y los objetivos finales de su forma de actuar.

Por lo tanto, la empresa tiene que comprometerse a formar a sus directivos, de forma que acepten el papel central de los valores incluso en el trabajo cotidiano, y además tiene que poseer sofisticadas herramientas de análisis para detectar, prevenir y afrontar concretamente los problemas éticos que influyen en su gestión, además de intentar alinear las políticas futuras con los principales valores emergentes dentro de la cultura ética de la sociedad.

Lo más importante es que tales valores definan los derechos y las obligaciones de los actores sociales, y por lo tanto, la conveniencia de tomar ciertas decisiones y de llevar a cabo ciertas acciones.

El análisis ético no sólo consiste en la referencia a un conjunto de normas rígido, sino en un esquema de razonamiento gestional orientado a generar un proceso lógicoracional de valoración acerca de la conveniencia de decisiones y comportamientos planteados por cualquier sujeto social, incluida la empresa.

Ante estas evidencias, parece clara la conveniencia de una nueva orientación de la cultura empresarial para superar la mera venta del producto, poner al cliente en el centro de la organización, pasar de ser una organización tecnology driver a ser customer focused.

Se trata de un desafío que en estos años han afrontado muchas organizaciones, pero sin ir más allá de la enunciación de los principios. En efecto, la dificultad mayor reside en traducir los principios en comportamientos eficaces y difundidos.

En tal sentido, la fidelity card representa la herramienta (el canal), a través de la cual la empresa puede influir en los elementos subyacentes de la confianza (los llamados drivers), y activar la relación con el cliente final. La tarjeta de fidelidad se convierte a todos los efectos en el vehículo de la relación fiduciaria entre el cliente y el distribuidor.

El proceso de desarrollo y consolidación de la relación fiduciaria con cada cliente sigue un camino que inicia con la introducción de la tarjeta y la recogida de un stock inicial de información. Los pasos siguientes que conciernen las acciones de loyalty management, son fundamentales para que el potencial generativo creado por la tarjeta sea activado efectivamente por la empresa. Ésto significa que, la tarjeta de fidelidad, es la herramienta de una relación de confianza solamente potencial si falta una actividad consciente orientada a explotar las oportunidades emergentes. 
Por lo tanto, el distribuidor debe estimular al consumidor a adoptar su tarjeta de fidelidad, siendo consciente de que la fidelización representa un objetivo estratégico a perseguir, aunque haya que sostener gastos significativos.

En la práctica, se trata de una empresa que sabe administrar su presente mientras planea e influencia el futuro y se distingue por estar customer oriented, es decir, centrada en el cliente, uno de los asset más críticos y estratégicos para la supervivencia de la empresa misma (Busacca, 2000).

La satisfacción del cliente sólo es el punto de partida para la construcción de una relación estable, pero no es suficiente, mientras que es esencial focalizar la atención en un factor que sin duda influye en el valor total de la empresa: la fidelidad de los clientes (customer loyalty). En efecto, a medida que aumente la customer satisfaction, y por lo tanto la confianza en la empresa, aumentará el grado de fidelidad de los clientes, su disponibilidad a reconocer un valor añadido a los productos adquiridos y a extender la relación a otras categorías de bienes y/o servicios recogidos en la cartera empresarial (Johnson y Gustafsson, 2003).

\section{BIBLIOGRAFÍA:}

Anselmi, L., Lattanzi, N. (2002): Elementi di management e dinamica aziendale. Andamenti, decisioni, risultati, Torino, Giappichelli.

Bertini, U. (1975): “'L'azienda come sistema cibernetico. Studi di ragioneria, organizzazione, tecnica economica “, en AA.VV, vol. LOS, Pisa, Cursi.

Busacca, A.G. (2000): L'impresa vincente. Come avvalersi dell'informazione per soddisfare il cliente e vincere le sfide del 2000, Milano, F. Angeli.

Castaldo, S., Bertozzi, P. (2000): Category Management. Creare valore per il consumatore, Milano, McGraw-Hill.

Castaldo, S., Cillo, P. (2001): “Le strategie di accrescimento delle risorse fiduciarie nel retailing: il ruolo delle carte fedeltà", en Industria \& Distribuzione, n. 1, Milano, Apogeo.

Catturi, G. (2003): L'azienda universale: l'idea forza, la morfologia e la fisiologia, Padova, Cedam.

Cavalieri, E. (2002): Economia ed etica aziendale, Torino, Giappichelli.

Fazzini, M. (2006): Aggregazioni, accordi e alleanze tra imprese, Milano, F. Angeli.

Ferrarsi Franceschi, R. (1984): Finalità dell'azienda e condizioni di funzionamento, Pisa, SEU.

Figini, M. (2003): Dare valore alle esigenze dei clienti e dei dipendenti dell'azienda con la customer satisfaction ed i gruppi di miglioramento aziendali, Milano, F. Angeli.

Fiume, R. (2000): "Riflessioni sul ruolo di visione e missione nell'economia delle aziende”, en Rivista Italiana di Ragioneria e di Economia Aziendale”, n5-6, Roma.

Gazzoni, F. (2003): Manuale di Diritto Privato, Napoli, Ed. ESI.

Giunta, F. (1995): Appunti di economia aziendale, Padova, Cedam. 
Invernizzi, G. (1999): Il sistema delle strategie a livello aziendale, Milano, McGrawHill.

Johnson, M.D., Gustafsson, A. (2003): Customer satisfaction. Un sistema integrato di valutazione e gestione per incrementare la soddisfazione del cliente, la fedeltà e il profitto, Milano, Guerini y Adjuntos.

Lugli, G., Pellegrini, L. (2002): Marketing distributivo, Torino, Utet.

Manaresi, A. (1999): "La competizione tra distributori per la relazione con il cliente: dalla gestione di relazioni al recupero del paradigma transazionale”, en Trade marketing, n. 26, Milano, F. Angeli.

Marra, A. (2002): L'etica aziendale come motore di progresso e di successo, Milano, F. Angeli.

Riolo, F. (1995): Etica degli affari e codici aziendali, Milano, Edibank.

Fazzini, M. (2006): Aggregazioni, accordi e alleanze tra imprese, Milano, F. Angeli.

Volpato, G. (2000): La gestione d'imprese $2^{\circ} \mathrm{ed}$., Padova, CEDAM.

Zappa, G. (1956): Le produzioni nell'economia delle imprese, Tomo I, Milano, Giuffrè. 